https://helda.helsinki.fi

Cost savings in outpatient versus inpatient reduction mammaplasty

Carpelan, A.

2016-11

Carpelan , A \& Kauhanen , S 2016 , ' Cost savings in outpatient versus inpatient reduction mammaplasty ' , Journal of Plastic, Reconstructive \& Aesthetic Surgery , vol. 69 , no. 11 , pp. 1486-1489 . https://doi.org/10.1016/j.bjps.2016.05.008

http://hdl.handle.net/10138/229849

https://doi.org/10.1016/j.bjps.2016.05.008

publishedVersion

Downloaded from Helda, University of Helsinki institutional repository.

This is an electronic reprint of the original article.

This reprint may differ from the original in pagination and typographic detail.

Please cite the original version. 


\title{
Cost savings in outpatient versus inpatient reduction mammaplasty
}

\author{
A. Carpelan ${ }^{a, b, *}$, S. Kauhanen ${ }^{a}$ \\ ${ }^{a}$ Department of Plastic and Reconstructive Surgery, Helsinki University Hospital Jorvi, Finland \\ ${ }^{\mathrm{b}}$ Department of Digestive Surgery, University of Turku and Turku University Hospital, Finland
}

Received 29 January 2016; accepted 17 May 2016

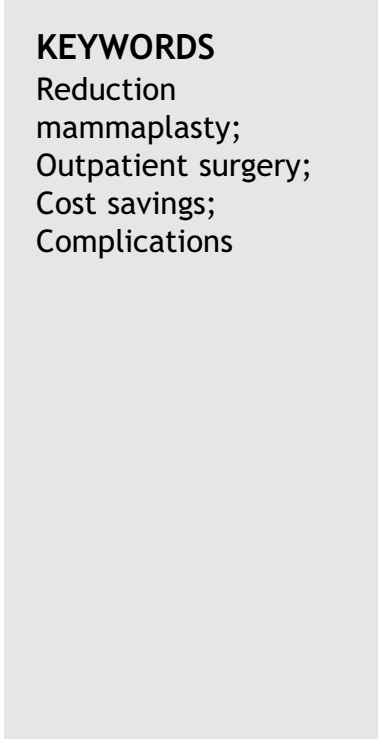

\begin{abstract}
Summary Background and aims: Reduction mammaplasties are increasingly performed as outpatient procedures. Cost savings are assumed, but published data on the subject are scarce. The aim of this study was to retrospectively determine the possible cost savings achieved by performing reduction mammaplasties as outpatient procedures.

Material and methods: Reduction mammaplasty was performed for 90 outpatients and 44 inpatients, with comparable health status. Demographic, surgical, and complication data were collected retrospectively. Data on the costs of the entire treatment process were acquired and statistical analyses performed.

Results: The average total cost of the process was $5039 €$ for inpatients and $4114 €$ for outpatients. Thus, the total costs were $925 €(18 \%)$ lower for the outpatient procedures. On average, cost saving per patient was $294 €(43 \%)$ on ward expenditures. Higher ward expenditure was a statistically significant cause of the increased cost of the inpatient group on uni- and multivariable analyses; however, for total costs, the effects of complications and reoperations were significant.

Conclusions: Reduction mammaplasty performed as an outpatient procedure results in up to $18 \%$ cost savings compared with inpatient treatment.

(C) 2016 British Association of Plastic, Reconstructive and Aesthetic Surgeons. Published by Elsevier Ltd. All rights reserved.
\end{abstract}

\footnotetext{
* Corresponding author. Department of Digestive Surgery, Turku University Hospital, P. O. Box 52, FI-20520 Turku, Finland. Tel.: $+35823135093$.

E-mail address: anu.carpelan@fimnet.fi (A. Carpelan).
}

\section{Introduction}

Although it is often assumed that outpatient procedures are less expensive than inpatient procedures, published reports on the subject are scarce. Costs involved in cataract surgery have been shown to be $20 \%$ higher for inpatients. ${ }^{1}$ Day case surgery for Dupuytren's contracture has been shown to be associated with $18 \%$ cost savings compared with 
inpatient treatment. ${ }^{2}$ In breast surgery, lumpectomy and mastectomy performed as outpatient procedures have been shown to result in cost savings of $75-78 \%$ compared with 2 or 3 days of hospitalization. ${ }^{3}$

To the best of our knowledge, studies specifically addressing the costs of outpatient reduction mammaplasties have not been published yet, although the issue has been briefly discussed in few previous articles. Buenaventura et al. ${ }^{4}$ reported cost savings of US $\$ 1500-2500$ (25-35\%) per patient, but this was based on estimated average costs of a reduction mammaplasty surgery in different facilities and not on direct calculations at the level of specific patients. Davies et al. ${ }^{5}$ reported cost savings of $40-50 \%$ compared with the average costs of outpatient $(n=20)$ and inpatient $(n=20)$ procedures, but without specifying the parameters included in the costs (except for excluding surgeon's fee). Short et al. ${ }^{6}$ found that outpatient procedures are 30\% less expensive than surgeries with a one-night hospital stay, although for longer stays the difference was even higher. However, in their study, the groups were not similar: the inpatients weighed more and had more complications, possibly increasing the costs of treatment. In addition, they did not specify the components of their cost analysis. Nelson et al. ${ }^{7}$ reported on practice profiles of $\mathrm{Ca}$ nadian plastic surgeons in reducing breast size. They mentioned that the day surgery fee was US $\$ 300$ and the cost of a 24-h ward admission was US \$1173, resulting in a cost saving of $75 \%$.

In the evaluation of the possible cost benefits of outpatient surgery, a number of different cost parameters have to be taken into account. In addition to the surgical procedure and stay in the outpatient unit or overnight in the hospital ward, the costs of pre- and postoperative planned and unplanned visits to the clinic and possible readmissions and reoperations have to be considered. This will reflect almost the costs of the entire process of surgical treatment of breast hypertrophy as an outpatient or inpatient procedure.

Outpatient breast reduction procedures were started in our university hospital outpatient unit in 2006. The purpose of this study was to evaluate the possible cost savings achieved by this change in practice.

\section{Material and methods}

Reduction mammaplasty patient records obtained from our university hospital outpatient unit were examined. The retrospective study covered a period between September 2006 and May 2009. Inclusion criteria were bilateral mammaplasties by either pedicled technique or free nipple grafting with the minimum resection weight of $400 \mathrm{~g}$. Procedures done on males for gynecomastia were excluded from the study. During the study period, some of the patients who were originally selected to have an outpatient procedure were reassigned to be treated as inpatients for nonclinical reasons (e.g., waiting time for an outpatient surgery exceeding 6 months). These patients were then allocated to the inpatient comparison group. Patients who were operated in the operating room of the outpatient unit and admitted overnight for social reasons (no adult to accompany the patient for the first $24 \mathrm{~h}$ postoperatively) were also transferred to the inpatient comparison group.

Medical records of all the eligible patients were reviewed and relevant demographic and surgical information were collected. All complications, reoperations, and unexpected hospital admissions and return visits together with their reasons were recorded. Complications were further defined as minor and major. Minor complications included delayed healing, superficial infections, and seromas, whereas major complications included wound dehiscence of $>2 \mathrm{~cm}$ lasting for $>4$ weeks after the surgery, hematomas that required evacuation, deep infections requiring drainage or intravenous antibiotics, and nipple or fat necrosis.

In our university hospital, information about all costs of hospital treatment of individual patients are routinely stored in Ecomed ${ }^{\circledR}$ clinical patient administration database (Datawell Ltd., Espoo, Finland). In order to include all costs of the treatment process, data of each patient from the database were viewed from 12 months before the date of surgery to 18 months postoperatively. Data of all costs relating to the treatment of breast hypertrophy or possible postoperative complications were collected.

Statistical analysis was performed with SPSS Statistics version 22.0 and $\mathrm{R}$ version 2.15.3. Chi square test, Fisher's exact test, and independent sample T-test were performed for univariate analysis as appropriate for each variable. A general linear model was built for univariate analysis between group effects. A multivariate analysis of variance (MANOVA) was performed to define demographic or surgical variables correlated with total cost, procedural cost, and ward expenditure. Statistical significance was set at $p<0.05$.

\section{Results}

During the study period, 90 patients were treated as outpatients and 44 as inpatients. The demographic data as well as type of surgery performed were similar in the outpatient and inpatient groups (Table 1). Drains and prophylactic anticoagulant therapy were used more often in the inpatient group. The complication rate was higher for the inpatients and they also needed additional visits to dressing clinic and emergency polyclinic (Table 2).

The average total costs of the treatment process for the outpatients and inpatients were 4114 and $5039 €$, respectively, with the difference being statistically significant $(p=0.036)$. The maximum difference between the groups was in ward expenditure, but the costs were higher for the inpatients in all of the recorded cost categories (Table 3). The most expensive part of the process was the surgical procedure, including theater time. The general linear model showed that treatment group was a statistically significant reason for higher ward expenditures $(p=0.01)$, but not total expenditure $(p=0.082)$ or procedural cost $(p=0.091)$. Multivariate analysis showed a significant correlation between expenditures and treatment group ( $p=0.014$, Wilk's lambda 0.912), emergency room (ER) visit ( $p=0.001$, Wilk's lambda 0.873$)$, additional wound 
Table 1 Demographic and surgical information of the outpatient and inpatient groups.

\begin{tabular}{|c|c|c|c|}
\hline Variable & Outpatients & Inpatients & $\mathrm{p}$ \\
\hline Mean age, years (range) & $43(16-67)$ & $44(18-69)$ & ns \\
\hline Mean body mass index (BMI), $\mathrm{kg} / \mathrm{m}^{2}$ (range) & $27(20-37)$ & $28(20-37)$ & ns \\
\hline Smokers, $\mathrm{n}(\%)$ & $15(17)$ & $9(20)$ & ns \\
\hline Previous medical condition, $\mathrm{n}(\%)^{\mathrm{a}}$ & $47(52)$ & $26(59)$ & ns \\
\hline Mean operative time, min (range) & $164(99-259)$ & $162(99-228)$ & ns \\
\hline Mean weight of tissue resected, $\mathrm{g}$ (range) & $1150(400-3119)$ & $1312(403-3142)$ & ns \\
\hline Mean perioperative bleeding, ml (range) & $176(50-550)$ & $249(50-800)$ & 0.005 \\
\hline Use of drains, $n(\%)$ & $17(19)$ & $26(59)$ & $<0.00001$ \\
\hline Use of prophylactic anticoagulant therapy, n (\%) & $3(3)$ & $19(43)$ & $<0.00001$ \\
\hline Operating surgeon a resident in training, $\mathrm{n}(\%)$ & $14(16)$ & $13(30)$ & ns \\
\hline Procedure type, n (\%) & & & ns \\
\hline Pedicular nipple transposition & $82(91)$ & $40(90)$ & \\
\hline Free nipple graft & $3(3)$ & $2(5)$ & \\
\hline Pedicular nipple transposition and liposuction & $5(6)$ & $2(5)$ & \\
\hline Incision, n (\%) & & & ns \\
\hline Inverted T & $49(54)$ & $24(55)$ & \\
\hline Vertical & $41(46)$ & $20(45)$ & \\
\hline
\end{tabular}

${ }^{a}$ Previous medical condition refers to any illness or disease other than breast hypertrophy.

treatment ( $p=0.004$, Wilk's lambda 0.892), immediate reoperation ( $p<0.001$, Wilk's lambda 0.545$)$, and late reoperation ( $p<0.001$, Wilk's lambda 0.847).

\section{Discussion}

In this study, outpatient reduction mammaplasty procedures were associated with significantly lower costs than inpatient procedures. Total costs were $925 €(18 \%)$ lower for the outpatient procedures. On average, ward expenditure of $294 €(43 \%)$ per patient was saved. The savings were not as high as previously reported, ${ }^{4-7}$ which might have been due to several reasons. For example, our multivariate analysis showed that in addition to treatment group, costs correlated closely with ER visits and additional wound treatments as well as reoperations. Previous studies have not included these additional or

Table 2 Complications and revisits.

\begin{tabular}{llll}
\hline Variable & \multicolumn{3}{l}{ Outpatients } \\
\hline ER visits n (\%) & $15(17)$ & $14(32)$ & 0.046 \\
Dressing clinic n (\%) & $17(19)$ & $16(36)$ & 0.027 \\
Complications n (\%) & & & 0.026 \\
$\quad$ Minor & $38(42)$ & $24(55)$ & \\
$\quad$ Major & $13(14)$ & $10(23)$ & \\
$\quad$ No & $39(43)$ & $10(23)$ & \\
Immediate reoperations n (\%) & & & ns \\
$\quad$ Hematoma evacuation & $5(6)$ & $1(2)$ & \\
$\quad$ Revision for infection & $1(1)$ & $1(2)$ & \\
Late reoperations n (\%) & & & $\mathrm{ns}$ \\
$\quad$ Dog ear excision & $20(22)$ & $12(27)$ & \\
$\quad$ Other & $12(13)$ & $9(20)$ & \\
\hline
\end{tabular}

later costs. As complications are more and their treatments are expensive, ${ }^{8}$ this may account for much of our difference with previous studies. Moreover, all of the previous studies are from the United States and Canada, and therefore there will have been differences in the pricing of surgical procedures and ward expenditure from those of Europe and Finland.

For the inpatient treatment group, higher ward expenditures were a statistically significant cause for the higher costs of treatment, but for total costs, the sequelae of major complications played a significant role when the entire treatment process is included in the analysis. In particular, reoperations (theater time) and prolonged hospital stays were expensive. The number of complications was higher in the inpatient group, which increased the cost difference between the treatment groups. In our previous study, where we analyzed the complication profile of outpatient reduction mammaplasty patients, ${ }^{9}$ only longer operative time correlated with an increasing number and severity of complications.

Table 3 Average costs.

\begin{tabular}{lrll}
\hline Variable & Outpatients & Inpatients & $\mathrm{p}$ \\
\hline Total cost $(€)$ & 4114 & 5039 & 0.036 \\
Clinic costs $(€)$ & 583 & 685 & $\mathrm{~ns}$ \\
Cost of the procedure $(€)$ & 2307 & 2658 & $\mathrm{~ns}$ \\
Ward expenditure $(€)$ & 390 & 684 & 0.001 \\
Laboratory tests $(€)$ & 65 & 79 & $\mathrm{~ns}$ \\
Pathology $(€)$ & 397 & 414 & $\mathrm{~ns}$ \\
Clinical procedures $(€)$ & 270 & 400 & $\mathrm{~ns}$ \\
Radiology $(€)$ & 101 & 118 & $\mathrm{~ns}$ \\
Unspecified $(€)$ & 1 & 1,59 & $\mathrm{~ns}$ \\
\hline
\end{tabular}


There was no difference in the amount of complications between patients operated by residents or certified plastic surgeons. In this study, the operative times of outpatients and inpatients were similar, but inpatients seemed to be more often operated by residents, although the difference was not statistically significant. Drains were seldom used for outpatients. Removal of drains is difficult to arrange once the patient is discharged. For inpatients, drains were used more often, and it is possible that they have acted as routes for contamination and infection, thus increasing the amount of complications. The risk of thromboembolic events was considered higher for the inpatients resting at their hospital beds and thus prophylactic anticoagulant therapy was used more often for inpatients. This might have resulted in more bleeding and thus more hematomas and wound infections. Reduction mammaplasty is a procedure with a rather high $(40-50 \%)$ complication rate $e^{10-12}$ and the complication rate in our study population was similar to that previously reported. Fortunately, most of the complications were minor, but the costs of their treatment are an inevitable part of the expenditure of surgical treatment of breast hypertrophy. In spite of these additional costs, the cost utility of reduction mammaplasty is high, as expressed by the quality-adjusted life years gained by the assets invested in the treatment process. ${ }^{13}$ The cost utility can be further increased with the savings achieved by outpatient treatment.

The major limitations of this study are its retrospective nature and the small sample size. On the contrary, as a retrospective study, we believe that it reflects the true costs in our outpatient and inpatient units. In spite of the limitations, we also believe that our study suggests that outpatient mammaplasty is cheaper than inpatient mammaplasty. On the basis of this study, we feel that outpatient reduction mammaplasty should be adopted more widely in Europe.

\section{Conflict of interest statement}

A. Carpelan: None.

S. Kauhanen: None.

\section{Acknowledgments}

The authors would like to thank MD Risto Roine for his guidance in the use of the Ecomed ${ }^{\circledR}$ database and MD Jarmo Gunn for statistical analyses.

\section{References}

1. Fedorowicz Z, Lawrence D, Gutierrez P, van Zuuren EJ. Day care versus in-patient surgery for age-related cataract. Cochrane Database Syst Rev 2011;6(7):CD004242.

2. Gerber RA, Perry R, Thompson R, Bainbridge C. Dupuytren's contracture: a retrospective database analysis to assess clinical management and costs in England. BMC Musuloskelet Disord 2011;12(12):73.

3. McManus SA, Topp DA, Hopkins C. Advantages of outpatient breast surgery. Am Surg 1994;60(12):967-70.

4. Buenaventura S, Severinac R, Mullis $W$, et al. Outpatient reduction mammaplasty: a review of 338 consecutive cases. Ann Plast Surg 1996;36(2):162-6.

5. Davies B, Lewis R, Pennigton G. Reduction mammaplasty: a comparison of outpatient and inpatient procedures. Aesthetic Plast Surg 1996;20:77-80.

6. Short K, Ringler S, Bengtson B, et al. Reduction mammaplasty: a safe and effective outpatient procedure. Aesthetic Plast Surg 1996;20:513-8.

7. Nelson RA, Colohan SM, Sigurdson LJ, Lalonde DH. Practice profiles in breast reduction: a survey among Canadian plastic surgeons. Can J Plast Surg 2008;16(3):157-61.

8. Nwaogu IY, Bommarito K, Olsen MA, Margenthaler JA. Economic impact of bleeding complications after mastectomy. J Surg Res 2015 Nov; 199(1):77-83.

9. Carpelan A, Kauhanen S, Mattila K, et al. Reduction mammaplasty as an outpatient procedure: a retrospective analysis of outcome and success rate. Scand J Surg 2015 Jun;104(2):96-102.

10. Cunningham BL, Gear AJL, Kerrigan CL, et al. Analysis of breast reduction complications derived from the BRAVO study. Plast Reconstr Surg 2005;115:1597-604.

11. Davis GM, Ringler SL, Short K, et al. Reduction mammaplasty: long-term efficacy, morbidity, and patient satisfaction. Plast Reconstr Surg 1995;96(5):1106-10.

12. Dabbah A, Lehman Jr JA, Parker MG, et al. Reduction mammaplasty: an outcome analysis. Ann Plast Surg 1995;35(4):337-41.

13. Tykkä $E$, Räsänen $P$, Tukiainen $E$, et al. Cost-utility of breast reduction surgery-a prospective study. J Plast Reconstr Aesthet Surg 2010 Jan;63(1):87-92. 